\title{
DOT PRODUCT REARRANGEMENTS
}

\author{
PAUL ERDOS and GARY WEISS \\ Mathematics Institute, Budapest; University of Cincinnati \\ (Received September 18, 1980)
}

ABSTRACT. Let $a=\left(a_{n}\right), x=\left(x_{n}\right)$ denote nonnegative sequences; $x=\left(x_{\pi(n)}\right)$ denotes the rearranged sequence determined by the permutation $\pi, a \cdot x$ denotes the dot product $\sum a_{n} x_{n}$; and $S(a, x)$ denotes $\left\{a \cdot x_{\pi}: \pi\right.$ is a permuation of the positive integers $\}$. We examine $S(a, x)$ as a subset of the nonnegative real line in certain special circumstances. The main result is that if $a_{n} \uparrow \infty$, then $S(a, x)=[a \cdot x, \infty]$ for every $x_{n} \neq 0$ if and only if $a_{n+1} / a_{n}$ is uniformly bounded.

KEY WORDS AND PHRASES. Dot product, series rearrangements, conditional convergence. 1982 MATHEMATICS SUBJECT CLASSIFICATION CODE. $40 A 05$.

An elementary classical result of Riemann on infinite series states that a conditionally convergent series that is not absolutely convergent can be rearranged to sum to any extended real number. A slightly similar group of questions arose in connection with certain formulas in operator theory $[1, p .181]$. Namely, if we let $a=\left(a_{n}\right)$, $x=\left(x_{n}\right)$ denote any two non-negative sequences and $x_{\pi}$ denote the sequence $\left(x_{\pi(n)}\right)$ where $\pi$ is any permutation of the positive integers, then what can be said about the set of non-negative real numbers $S(a, x)=\{a \cdot x: \pi$ is a permutation of the positive lintegers\}. More specifically, which subsets of the non-negative real line can be realized as the form $S(a, x)$ for some such $a$ and $x$ ?

Various facts about $S(a, x)$ are obvious 
(1) $\mathrm{s}(\mathrm{a}, \mathrm{x}) \subset[0, \infty]$. The values 0 and $\infty$ may be obtained.

(2) If $a$ and $x$ are strictly positive sequences or are at most finitely zero, then $s(a, x) \subset(0, \infty]$

(3) Not all subsets of $[0, \infty]$ are realizable as an $s(a, x)$ set. This follows by a cardinality argument. If $c$ denotes the cardinality of $[0, \infty]$, then the cardinality of the class of subsets of $[0, \infty]$ is $2^{c}$, but the cardinality of the class of sequences $a$ and $x$ is $c$ and thus the cardinality of the subsets $S(a, x)$ is less than or equal to $c \cdot c=c$.

(4) If either $a$ or $x$ is finitely non-zero then $S(a, x)$ is countable.

(5) An example: if $a=(0,2,0,2, \ldots)$ and $x=\left(3^{-n}\right)$, then $s(a, x)$ is precisely the Cantor set except for those non-negative real numbers whose ternary expansion consists of a tail of $0^{\prime}$ s or a tail of 2's (i.e., a subset of the rational numbers.),

It seems too ambitious to consider the general question at this time. For this reason we shall restrict our attention to the cases when a is a non-decreasing sequence and $\mathbf{x}$ is a non-increasing sequence,

If $a \equiv 0$ or $x \equiv 0$, the problem is trivial and $s(a, x)=\{0\}$. If $a_{1} \neq 0$ and $x_{n} \nrightarrow 0$, the problem is trivial and $S(a, x)=\{\infty\}$. If $a_{n}$ is bounded by $M$, then $S(a, x) \subset\left[0, M\left[x_{n}\right]\right.$. In any case, hereafter we shall assume $a_{n} \uparrow \infty$ and $x_{n} \downarrow 0$, unless otherwise specified.

The Lemma that follows is a well-known fact, but we give a proof for completeness and because the proof contains some of the ideas used in the main result.

LEMMA. If $a_{n} \uparrow$ and $x_{n} \downarrow$ then $s(a, x) \subset[a \cdot x, \infty]$. In addition, $a \cdot x \in S(a, x)$, and if $a_{n} \uparrow \infty$ and $x_{n} \neq 0$ for $a l l n$ or if $a_{n} \uparrow$ and $a_{n}>0$ for some $n$ and $x_{n} \neq 0$, then $\infty \varepsilon S(a, x)$.

PROOF. It suffices to show that for every permutation $\pi$ of the positive integers, we have $a \cdot x \leq \sum a_{n} x_{\pi(n)}$ or, equivalently, $a \cdot x \leq \sum a_{\pi(n)} x_{n}$ for every $\pi$. The rest of the lemma is clear.

Define $\pi_{1}$ in terms of $\pi$ as follows. Set 


$$
\pi_{1}(n)=\left\{\begin{array}{cl}
1 & n=1 \\
\pi(1) & n=\pi^{-1}(1) \\
\pi(n) & \text { otherwise }
\end{array}\right.
$$

It is straightforward to verify that $\pi_{1}$ is also a permutation of the positive integers (one-to-one and onto) which fixes 1 . We assert that $a_{\pi} \cdot x \leq a_{\pi} \cdot x$ To see this, note that $\pi(1) \geq 1$ and $\pi^{-1}(1) \geq 1$. Hence $a_{\pi(1)}-a_{1} \geq 0$ and $x_{1}-x_{\pi^{-1}} \geq 0$. Therefore

$$
\begin{aligned}
\sum\left(a_{\pi(n)}-a_{\pi_{1}(n)}\right) x_{n} & =\left(a_{\pi(1)}-a_{\pi_{1}(1)}\right) x_{1}+\left(a_{\pi\left(\pi^{-1}(1)\right)}-a_{\pi_{1}}\left(\pi^{-1}(1)\right) x_{\pi}^{-1}(1)\right. \\
& =\left(a_{\pi(1)}-a_{1}\right)\left(x_{1}-x_{\pi}^{-1}\right) \\
& \geq 0 .
\end{aligned}
$$

Proceeding inductively, we obtain a sequence of permutations $\pi_{k}$ that $f i x l, 2, \ldots, k$ for which $a_{\pi_{k}} \cdot x \leq a_{\pi_{k-1}} \cdot x$. Hence, for every $k$,

$$
\sum_{n=1}^{k} a_{n} x_{n}=\sum_{n=1}^{k} a_{\pi_{k}}(n) x_{n} \leq a_{\pi_{k}} \cdot x \leq a_{\pi} \cdot x
$$

Letting $\mathrm{k} \rightarrow \infty$, we obtain $\mathrm{a} \cdot \mathrm{x} \leq \mathrm{a}_{\pi} \cdot \mathrm{x}$.

The main question of this paper is: for which $a, x$ with $a_{n} \uparrow \infty$ and $x_{n} \downarrow 0$

is $S(a, x)=[a \cdot x, \infty]$ ?

The main result of this paper gives a partial answer. Namely, we can characterize which $a_{n} \uparrow \infty$ have the property that $S(a, x)=[a \cdot x, \infty]$ for every $x$ such that $x_{n} \neq 0$.

On first sight, it might appear that $s(a, x)$ can never be $[a \cdot x, \infty]$ or that it is quite rare. The first result in this direction was that if $a_{n}=n$ for every $n$, then $S(a, x)=[a \cdot x, \infty]$ for every $x$ such that $x_{n} \neq 0$. That $s(a, x)$ may not be $[a \cdot x, \infty]$ was first decided by an example due to Robert Young. Namely, let $a_{n}=2^{2^{n}}$ and $x_{n}=2^{-2^{n+1}}$. Both results are unpublished. The succeeding results and techniques are due to the work of the authors in collaboration with Hugh Montgomery. 
THEOREM 1. (The Main Theorem) Let $a=\left(a_{n}\right)$ where $a_{n}>0$ for every $n$ and $a_{n} \rightarrow \infty$. Consider the following conditions:

(1) $a_{n+1} / a_{n}$ is bounded.

(2) For the non-negative sequence $x=\left(x_{n}\right)$, there exist subsequences $\left(a_{n_{k}}\right)$ and $\left(\mathrm{x}_{\mathrm{m}_{\mathrm{k}}}\right)$ of $\mathrm{a}$ and $\mathrm{x}$ respectively such that

(a) $a_{n_{k}} x_{m_{k}} \rightarrow 0$ as $k \rightarrow \infty$, and

(b) $\sum_{\mathrm{k}} \mathrm{a}_{\mathrm{n}_{\mathrm{k}}} \mathrm{x}_{\mathrm{m}_{\mathrm{k}}}=\infty$.

(3) $\quad S(a, x)=[a \cdot x, \infty]$.

Then (1) implies (2) for every strictly positive sequence $x=\left(x_{n}\right)$ that tends to 0 . Also if $a_{n} \uparrow \infty$ and $x_{n} \downarrow 0$ where $a_{n}, x_{n} \neq 0$ for all $n$, then (2) implies (3).

PROOF. To prove that (1) implies that (2) holds for every strictly positive sequence $x=\left(x_{n}\right)$ that tends to 0 , suppose $a_{n+1} / a_{n} \leq M$ for all $n$. We assert that for every positive integer $k$, there exist arbitrarily large positive integers $\mathrm{n}_{\mathrm{k}}$ and $\mathrm{m}_{\mathrm{k}}$ for which $(\mathrm{k}+1)^{-1} \leq \mathrm{a}_{\mathrm{n}_{\mathrm{k}}} \mathrm{x}_{\mathrm{m}_{\mathrm{k}}} \leq \mathrm{Mk}^{-1}$. If this assertion were true, then clearly we could choose two strictly increasing subsequences of positive integers $\left(n_{k}\right)$ and $\left(m_{k}\right)$ such that $a_{n_{k}} x_{k} \rightarrow 0$ as $k \rightarrow \infty$ to prove the assertion.

For each fixed positive integer $k,(k+1)^{-1} \leq a_{n} x_{m} \leq k^{-1}$ if and only if $x_{m} \varepsilon\left[\left(a_{n}(k+1)\right)^{-1}, M\left(a_{n} k\right)^{-1}\right]$. All we need show is that there exist arbitrarily large $n, m$ for which $x_{m} \varepsilon\left[\left(a_{n}(k+1)\right)^{-1}, M\left(a_{n} k\right)^{-1}\right]$.

Suppose to the contrary that there exists a positive integer $\mathrm{N}$ for which $x_{m} \notin\left[\left(a_{n}(k+1)\right)^{-1}, M\left(a_{n} k\right)^{-1}\right]$ for every $n, m \geq N$. In other words, for every $m \geq N$, $x_{m} \notin \bigcup_{n \geq N}\left[\left(a_{n}(k+1)\right)^{-1}, M\left(a_{n} k\right)^{-1}\right]$. (Note: This would imply that $\bigcup_{n \geq N}\left[\left(a_{n}(k+1)\right)^{-1}\right.$, $\left.M\left(a_{n} k\right)-1\right]$ cannot contain any interval of the form $(0, \varepsilon)$ for some $\varepsilon>0$, since $x_{m} \rightarrow 0$ as $m \rightarrow \infty$. However, this is not the case. Indeed, the proof below can be used to show that for every $N$, there exists $\varepsilon>0$ such that $\left.(0, \varepsilon) \subset \bigcup_{n \geq N}\left[\left(a_{n}(k+1)\right)^{-1}, M\left(a_{n} k\right)^{-1}\right].\right)$.

For each $m \geq N$, let $n_{m}$ denote the least positive integer $n$ such that $M\left(a_{n+1} k\right)^{-1}<x_{m}$, which exists since $a_{n} \rightarrow \infty$ as $n \rightarrow \infty$ and hence $M\left(a_{n+1} k\right)^{-1} \rightarrow 0$ 
as $n \rightarrow \infty$. For $m$ sufficiently large, we have $M\left(a_{n_{m}}+l^{k)^{-1}} \leq x_{m} \leq M\left(a_{n_{k}} k\right)^{-1}\right.$. Also, since $M\left(a_{n_{m}} k\right)^{-1}<x_{m}$ and $x_{m} \rightarrow 0$ as $m \rightarrow \infty$, we have $m \rightarrow \infty$ implies $a_{n_{m}+1} \rightarrow \infty$ and hence $n_{m} \rightarrow \infty$. Therefore $n_{m} \geq N$ for all $m$ sufficiently large, and for these $m, x_{m} \notin\left[\left(a_{n_{m}}(k+1)\right)^{-1}, M\left(a_{n_{m}} k\right)^{-1}\right]$. Hence, for infinitely many $m$, we have $x_{m} \leq M\left(a_{n_{k}} k\right)^{-1}$ and $x_{m} \notin\left[\left(a_{n_{m}}(k+1)\right)^{-1}, M\left(a_{n_{m}} k\right)^{-1}\right]$. Therefore, for infinitely many $m$, we have $M\left(a_{n_{m}+1} k\right)^{-1}<x_{m}<\left(a_{n_{m}}(k+1)\right)^{-1}$. This implies that $M\left(a_{n_{m}} k\right)^{-1}<\left(a_{n_{m}}(k+1)\right)^{-1}$ for infinitely many $m$, or equivalently, $a_{n}+1 / a_{m}>M(k+1) / k>M$ for infinitely many $m$, which contradicts our assumption that $a_{n+1} / a_{n} \leq M$ for all $n$. Hence (2) is proved.

To prove $(2) \rightarrow(3)$ whenever $a_{n} \uparrow \infty$ and $x_{n \neq} 0$, suppose (2) holds for a and $x$, so that there exist subsequences $\left(a_{n_{k}}\right)$ and $\left(x_{m_{k}}\right)$ such $a_{n_{k}} x_{m_{k}} \rightarrow 0$ as $k \rightarrow \infty$, and $\sum_{k} a_{n_{k}} x_{k}=\infty$. We first assert that without loss of generality we may assume that $a \cdot x=\sum a_{n} x_{n}<\infty$. To see this suppose $a \cdot x=\sum a_{n} x_{n}=\infty$. Then by the lemma we have that $S(a, x)=\{\infty\}$, and hence (3) holds.

Assuming that $\sum a_{n} x_{n}<\infty$, we next assert that without loss of generality we can assume that $\mathrm{n}_{\mathrm{k}}>\mathrm{m}_{\mathrm{k}}$ for every $\mathrm{k}$. To see this, let $\mathrm{z}_{\mathrm{l}}$ denote the set $\left\{\mathrm{k}: \mathrm{n}_{\mathrm{k}}>\mathrm{m}_{\mathrm{k}}\right\}$ and let $\mathrm{z}_{2}$ denote the set $\left\{\mathrm{k}: \mathrm{n}_{\mathrm{k}} \leq \mathrm{m}_{\mathrm{k}}\right\}$. Then

$$
\infty=\sum_{k} a_{n_{k}} x_{m_{k}}=\sum_{k \in z_{1}} a_{n_{k}} x_{m_{k}}+\sum_{k \varepsilon z_{2}} a_{n_{k}} x_{m_{k}}
$$

But $\sum_{k \in z_{2}} a_{n_{k}} x_{m_{k}} \leq \sum_{k \in z_{2}} a_{n_{k}} x_{n_{k}} \leq \sum_{n} a_{n} x_{n}<\infty$. Therefore $\underset{k \varepsilon z_{l}}{\sum_{k}} a_{n_{k}} x_{m_{k}}=\infty$ Let $z_{1}$ determine subsequences of $\left(n_{k}\right)$ and $\left(m_{k}\right)$, whlch for simplicity we again call ( $\left.n_{k}\right)$ and $\left(m_{k}\right)$, respectively, by taking only those entries $n_{k}, m_{k}$ (in increasing order) for which $\mathrm{k} \in \mathrm{E}_{\mathrm{l}}$. This gives us subsequences $\left(\mathrm{a}_{\mathrm{n}_{\mathrm{k}}}\right)$ and $\left(\mathrm{x}_{\mathrm{m}_{\mathrm{k}}}\right)$ of $\mathrm{a}$ and $\mathrm{x}$ which satisfy conditions $a$ and $b$ in the $2^{\text {nd }}$ condition of the theorem, and in addition satisfy $\mathrm{n}_{\mathrm{k}}>\mathrm{m}_{\mathrm{k}}$ for all $\mathrm{k}$.

Next we assert that without loss of generality we may assume $n_{k} \neq m_{j}$ for all $k, j$. To see this, note that we have $n_{k}>m_{k}$ for all $k$ and that $\left\langle n_{k}>\right.$ and $<m_{k}>$ are strictly increasing (a property of subsequences). Therefore if $\mathrm{n}_{\mathrm{k}}=\mathrm{m}_{\mathrm{j}}$ for 
some $k, j$, then $k<j$ and $n_{k} \neq m_{i}$ for all $i \neq j$. That is, $n_{k}$ can occur at most once among the $m_{j}$ s. Put $\left(n_{1}, m_{1}\right), \ldots,\left(n_{k_{1}}, m_{k_{1}}\right) \varepsilon s_{l}$ where $k_{1}+1$ is the least positive integer such that $\mathrm{m}_{\mathrm{k}_{1}+1}=\mathrm{n}_{\mathrm{k}}$ for some $\mathrm{k}<\mathrm{k}_{1}+1$. Put $\left(\mathrm{n}_{\mathrm{k}_{1}+1}, \mathrm{~m}_{\mathrm{k}_{1}+1}\right), \ldots,\left(\mathrm{n}_{\mathrm{k}_{2}}, \mathrm{~m}_{\mathrm{k}_{2}}\right) \in \mathrm{S}_{2}$ where $\mathrm{k}_{2}+1$ is the least positive integer, if it exists, such that $m_{k_{2}+1}=n_{k}$ for some $k_{1}+1 \leq k<k_{2}+1$. Put $\left(n_{k_{2}+1}, m_{k_{2}+1}\right), \ldots$, $\left(\mathrm{n}_{\mathrm{k}_{3}}, \mathrm{~m}_{\mathrm{k}_{3}}\right) \varepsilon \mathrm{s}_{1}$ such that $\mathrm{k}_{3}+1$ is the least positive integer, if it exists, such that $\mathrm{m}_{\mathrm{k}_{3}+1}=\mathrm{n}_{\mathrm{k}}$ for some $\mathrm{k} \leq \mathrm{k}_{1}$ or $\mathrm{k}_{2} \leq \mathrm{k}<\mathrm{k}_{3}+1$. Continuing in this way, if no such least positive integer exists, then either $s_{1}$ or $s_{2}$ is finite. Otherwise both $s_{1}, s_{2}$ are infinite. For either case, no $n_{k}=m_{j}$ when both $\left(n_{k}, m_{k}\right),\left(n_{j}, m_{j}\right)$ $\varepsilon S_{1}$ or $S_{2}$. Then clearly $S_{1}, S_{2}$ is a disjoint partition of the set of all $\left(n_{k}, m_{k}\right)$ and in each set, no $\mathrm{n}_{\mathrm{k}}$ appears as an $\mathrm{m}_{\mathrm{j}}$. Therefore $\infty=\sum \mathrm{a}_{\mathrm{n}_{\mathrm{k}}} \mathrm{x}_{\mathrm{m}_{\mathrm{k}}}=\sum_{\mathrm{S}_{1}} \mathrm{a}_{\mathrm{n}_{\mathrm{k}}} \mathbf{x}_{\mathrm{m}_{\mathrm{k}}}+$ $+\sum_{\mathrm{S}_{2}} \mathrm{a}_{\mathrm{n}_{\mathrm{k}}} \mathrm{x}_{\mathrm{m}_{\mathrm{k}}}$, and so either $\sum_{\mathrm{s}_{1}} \mathrm{a}_{\mathrm{n}_{\mathrm{k}}} \mathrm{x}_{\mathrm{m}_{\mathrm{k}}}=\infty \quad$ or $\sum_{\mathrm{s}_{2}} \mathrm{a}_{\mathrm{n}_{\mathrm{k}}} \mathrm{x}_{\mathrm{m}_{\mathrm{k}}}=\infty$. Choosing $\mathrm{s}_{1}$ or $\mathrm{S}_{2}$ accordingly we produce the sequence $\left(\mathrm{n}_{\mathrm{k}}, \mathrm{m}_{\mathrm{k}}\right)$ with the desired properties, (i.e., satisfying a) and $b$ ) in Theorem $l$ and also satisfying $n_{k} \neq m_{j}$ for all $k, j$ and $\mathrm{n}_{\mathrm{k}}>\mathrm{m}_{\mathrm{k}}$ for every $\mathrm{k}$.).

Now consider the series $\sum_{k}\left(a_{n_{k}}-a_{m_{k}}\right)\left(x_{m_{k}}-x_{n_{k}}\right)$. Since $n_{k}>m_{k}$, we have $0 \leq a_{n_{k}}-a_{m_{k}} \leq a_{n_{k}}$ and $0 \leq x_{m_{k}}-x_{n_{k}} \leq x_{m_{k}}^{\prime}$ and so $0 \leq\left(a_{n_{k}}-a_{m_{k}}\right)\left(x_{m_{k}}-x_{n_{k}}\right)$ $\leq a_{n_{k}} x_{m_{k}} \rightarrow 0$ as $k \rightarrow \infty$. Furthermore, since $\sum_{k} a_{n_{k}} x_{m_{k}}=\infty, a_{m_{k}} x_{n_{k}} \geq 0, \sum_{k} a_{n_{k}} x_{n_{k}}$

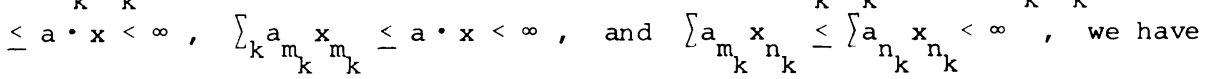

$$
\sum_{k}\left(a_{n_{k}}-a_{m_{k}}\right)\left(x_{m_{k}}-x_{n_{k}}\right)=\sum_{k}\left(a_{n_{k}} x_{m_{k}}+a_{m_{k}} x_{n_{k}}-a_{n_{k}} x_{n_{k}}-a_{m_{k}} x_{m_{k}}\right)
$$

We shall now show that for every $\varepsilon>0$, there exists a subsequence $\left(k_{n}\right)$ of positive integers such that $\varepsilon=\sum_{k \in\left\{k_{n}\right\}}\left(a_{n_{k}}-a_{m_{k}}\right)\left(x_{m_{k}}-x_{n_{k}}\right)$. This follows from the following more general fact.

Suppose $(d(k))$ is a non-negative sequence for which $d(k) \rightarrow 0$ as $k \rightarrow \infty$ and $\sum \mathrm{d}(\mathrm{k})=\infty$. We assert that very every $\varepsilon>0$, there exists a subsequence ( $\left.\mathrm{n}_{n}\right)$ such that $\varepsilon=\left[\mathrm{d}\left(\mathrm{k}_{\mathrm{n}}\right)\right.$. The proof of this fact proceeds along the same lines as the proof of Riemann's theorem on rearrangments of conditionally convergent series. Fix 
$\varepsilon>0$ and choose $\mathrm{n}_{1} \geq \mathrm{N}_{1}$ so that $\mathrm{d}(\mathrm{k})<\varepsilon$ for every $\mathrm{k} \geq \mathrm{N}_{1}$, and so that $\mathrm{n}_{1}$ is the greatest integer greater than $N_{1}$ such that $\sum_{k=N_{1}}^{n_{1}} d(k)<\varepsilon$. Hence $\sum_{k=N_{1}}^{n_{1}} d(k)<\varepsilon \leq \sum_{k=N_{1}}^{n_{1}^{+1}} d(k)$. This can be done since $d(k) \rightarrow 0$ as $k \rightarrow \infty$ and $d(k)=\infty$. Choose $\mathrm{N}_{2}>\mathrm{n}_{1}$ so that $\mathrm{d}(\mathrm{k})<\left(\varepsilon-\sum_{\mathrm{k}=\mathrm{N}_{1}} \mathrm{~d}(\mathrm{k})\right) / 2$ for every $\mathrm{k} \geq \mathrm{N}_{2}$ and then choose $\mathrm{n}_{2}$ to be the largest integer greater than $\mathrm{N}_{2}$ such that $\sum_{\mathrm{n}=\mathrm{N}_{2}}^{2} \mathrm{~d}(\mathrm{k})<\varepsilon-$ $\sum_{k=N_{1}}^{n_{1}} d(k)$. Hence $\sum_{k=N_{2}}^{n_{2}} d(k)<\varepsilon-\sum_{k=N_{1}}^{n_{1}} d(k) \leq \sum_{k=N_{2}}^{n_{2}+1} d(k)$. Proceeding inductively in this way, we obtain sequences $\left(N_{p}\right)$ and $\left(n_{p}\right)$ of positive integers for which $\mathrm{n}_{\mathrm{p}} \geq \mathrm{N}_{\mathrm{p}}>\mathrm{n}_{\mathrm{p}-1}, 0 \leq \mathrm{d}(\mathrm{k}) \leq\left(\varepsilon-\sum_{\mathrm{q}=1}^{\mathrm{p}-1} \sum_{\mathrm{k}=\mathrm{N}}^{\mathrm{n}} \mathrm{n}(\mathrm{k})\right) / 2^{\mathrm{p}-1}$ for every $\mathrm{p}$ and every $\mathrm{k} \geq \mathrm{N}_{\mathrm{p}}$, and

$$
\sum_{k=N_{p}}^{n} d(k)<\varepsilon-\sum_{q=1}^{p-1} \sum_{k=N}^{n} d(k) \leq \sum_{k=N}^{n} d(k) .
$$

This implies that

$$
\begin{aligned}
& 0<\varepsilon-\sum_{\mathrm{q}=1}^{\mathrm{p}} \sum_{k=\mathrm{N}}^{\mathrm{n} q} \mathrm{~d}(\mathrm{k}) \leq \mathrm{d}\left(\mathrm{n}_{\mathrm{p}}+1\right) \leq\left(\varepsilon-\sum_{\mathrm{q}=1}^{\mathrm{p}-1} \sum_{k=\mathrm{N}}^{\mathrm{n}} \mathrm{q}(\mathrm{k})\right) / 2^{\mathrm{p}-1} \\
& \leq \varepsilon / 2^{\mathrm{p}-1} \rightarrow 0 \text { as } \mathrm{p} \rightarrow \infty \text {. }
\end{aligned}
$$

Therefore $\varepsilon=\sum_{\mathrm{q}=1}^{\infty} \sum_{\mathrm{k}=\mathrm{N}}^{\mathrm{q}} \mathrm{d}(\mathrm{k})$. Hence, if we choose $\left(\mathrm{k}_{\mathrm{n}}\right)$ to be the strictly increasing sequence of positive integers $k$, where $k$ is taken to range over the set $\bigcup_{p=1}^{\infty}\left\{k: N_{p} \leq k \leq n_{p}\right\}$, we have $\varepsilon=\left[d\left(k_{n}\right)\right.$.

Applying this result to the sequence $\left(a_{n_{k}}-a_{m_{k}}\right)\left(x_{m_{k}}-x_{n_{k}}\right)$, since it is nonnegative, tends to 0 , and sums to $\infty$, we obtain that for every $\varepsilon>0$, there exist subsequences of $\left(n_{k}\right)$ and $\left(m_{k}\right)$, which we shall again denote by $\left(n_{k}\right)$ and $\left(m_{k}\right)$, for which $\varepsilon=\sum_{k}\left(a_{n_{k}}-a_{m_{k}}\right)\left(x_{m_{k}}-x_{n_{k}}\right)$.

Now recall that we wish to show that $S(a, x)=[a \cdot x, \infty]$. We already know $a \cdot x$ and $\infty \varepsilon S(a, x)$. Suppose $a \cdot x<r<\infty$. It suffices to show $r \varepsilon S(a, x)$. Let $\varepsilon=r-a \cdot x$ and choose subsequences which we again call $\left(n_{k}\right)$ and ( $\left.m_{k}\right)$ so that 


$$
\varepsilon=\sum_{k}\left(a_{n_{k}}-a_{m_{k}}\right)\left(x_{m_{k}}-x_{n_{k}}\right) .
$$

We now choose $\pi$, the requisite permutation on $z^{+}$, as follows. Let $\pi\left(n_{k}\right)=m_{k}$ and $\pi\left(m_{k}\right)=n_{k}$ for each $k$, and let $\pi$ fix all other integers $n$ (i.e., those $\mathrm{n}$ for which $\mathrm{n} \neq \mathrm{n}_{\mathrm{k}}, \mathrm{m}_{\mathrm{k}}$ for every $\left.\mathrm{k}\right)$. The permutation $\pi$ is well-defined since $n_{i} \neq m_{j}$ for every $i, j$. Let $z_{\pi}$ denote the set $\left\{n: n=n_{k}\right.$ or $n=m_{k}$ for some $\mathrm{k}\}$. Hence $\pi(\mathrm{n})=\mathrm{n}$ for all $\mathrm{n} \notin \mathrm{z}$. Then

$$
\begin{aligned}
\sum_{n} a_{n} x_{\pi(n)} & =\sum_{n \notin z} a_{n} x_{n}+\sum_{k}\left(a_{n_{k}} x_{m_{k}}+a_{m_{k}} x_{n_{k}}\right) \\
& \left.=\sum_{n \not z} a_{n} x_{n}+\sum_{k}\left(a_{n_{k}} x_{n_{k}}+a_{m_{k}} x_{m_{k}}\right)+\left(a_{n_{k}}-a_{m_{k}}\right)\left(x_{m_{k}}-x_{n_{k}}\right)\right) \\
& =\sum_{n} a_{n} x_{n}+\sum_{k}\left(a_{n_{k}}-a_{m_{k}}\right)\left(x_{m_{k}}-x_{n_{k}}\right) \\
& =a \cdot x+\varepsilon=r .
\end{aligned}
$$

and so $r \in S(a, x)$, which proves (3). Q.E.D.

THEOREM 2. Let $a=\left(a_{n}\right)$ where $a_{l}>0$ and $a_{n} \uparrow \infty$. Then $a_{n+l} / a_{n}$ is bounded if and only if, for every $x=\left(x_{n}\right)$ for which $x_{n \neq} 0, s(a, x)=[a \cdot x, \infty]$.

PROOF. If $a_{n+1} / a_{n}$ is bounded, then by Theorem 1 , if $x_{n \neq} 0$, then $x_{=}\left(x_{n}\right)$ satisfies condition (2) of the theorem. Also by Theorem 1 , since $a_{n} \uparrow \infty$ and $a_{1}>0$, condition (3) of the theorem is satisfied by $x$. That is, $S(a, x)=[a \cdot x, \infty]$.

Conversely, if $S(a, x)=[a \cdot x, \infty]$ for every $x=\left(x_{n}\right)$ for which $x_{n} \neq 0$, we claim that $a_{n+1} / a_{n}$ must remain bounded.

Suppose to the contrary that $a_{n+1} / a_{n}$ is not bounded. Let $h(n)$ denote the least positive integer $k$ for which $k \geq n$ and $a_{k+1} / a_{k} \geq 4^{n}$. Clearly $h(n)$ is a non-decreasing function of $n$. Define $x_{n}=\left(a_{h(n)} 3^{n}\right)^{-1}$. Then $x_{n} \neq 0$. Letting $x=\left(x_{n}\right)$, we claim that $s(a, x) \neq[a \cdot x, \infty]$. In fact, we claim that $a \cdot x<1$ but

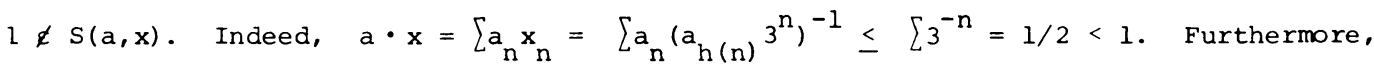
letting $\pi$ be any permuation of $z^{+}$, if $\pi^{-1}(k)>h(k)$ for some $k$, then

$$
\begin{aligned}
\sum a_{n} x_{\pi(n)} \geq a_{\pi^{-1}(k)} x_{k} & \geq a_{h(k)+1} x_{k}=a_{h(k)+1}\left(a_{h(k)} 3^{k}\right)^{-1} \\
& \geq 4^{k} 3^{-k}>1 .
\end{aligned}
$$


On the other hand, if $\pi^{-1}(k) \leq h(k)$ for every $k$, then

$$
\sum a_{n} x_{\pi(n)}=\sum a_{\pi-1} x_{(n)} \leq a_{h(n)} x_{n}=\int 3^{-n}=1 / 2<1 .
$$

In any case, $\sum a_{n} x_{\pi(n)} \neq 1$, hence $1 \not S(a, x) . \quad$ Q.E.D.

NOTE. In the proof of Theorem 1, each time we constructed a permutation $\pi$ to solve the equation $\sum a_{n} x_{\pi(n)}=r$, it sufficed to use only disjoint 2-cycles. That is, each such $\pi$ that we constructed was the product of disjoint 2-cycles. This seems odd and leads us to ask if there are any circumstances in which the use of infinite-cycles or n-cycles yields more. In other words, is it always true that $S(a, x)$ is the same as $\left\{\sum_{n} x_{\pi(n)}: \pi\right.$ is a permutation of $z^{+}$which is a product of disjoint 2-cycles\} ?

The following question seems likely to have an affirmative answer. If so, this would give a characterization for those sequences $a$ and $x$ where $a_{n} \uparrow \infty_{,} a_{1}>0$, and $x_{n} \neq 0$, which satisfy $s(a, x)=[a \cdot x, \infty]$. However, it remains unsolved.

QUESTION 1. If $a$ and $x$ are as above, does (3) $\Rightarrow(2)$ in Theorem 1?

Finally, we wish to point out that Theorems 1 and 2 imply analogous theorems in which $a$ and $x$ switch roles. Indeed, the proofs of the following two corollaries follow naturally along the same lines as those of Theorems 1 and 2 .

COROLLARY 3. Let $\mathbf{x}=\left(\mathrm{x}_{\mathrm{n}}\right)$ where $\mathrm{x}_{\mathrm{n}}>0$ for all $\mathrm{n}$, and $\mathrm{x}_{\mathrm{n}} \rightarrow 0$ as $\mathrm{n} \rightarrow \infty$. Consider the following conditions.

(1) $x_{n} / x_{n+1}$ is bounded below.

(2) For the non-negative sequence $a=\left(a_{n}\right)$, there exist subsequences $\left(a_{n_{k}}\right)$ and $\left(\mathrm{x}_{\mathrm{m}_{\mathrm{k}}}\right)$ of $\mathrm{a}$ and $\mathrm{x}$, respectively, such that
a) $a_{n_{k}} x_{m_{k}} \rightarrow 0$ as $k \rightarrow \infty$, and
b) $\quad \sum_{k} a_{n_{k}} x_{m_{k}}=\infty$.

Then (1) implies that (2) holds for every strictly positive sequence $a=\left(a_{n}\right)$ that tends to $\infty$. 
COROLLARY 4. Let $x=\left(x_{n}\right)$ be a non-negative sequence. Then $x_{n} / x_{n+1}$ is bounded below if and only if, for every $a=\left(a_{n}\right)$ for which $a_{n} \uparrow \infty$ and $a_{1}>0$, $S(a, x)=[a \cdot x, \infty]$

QUESTION 2. Is there anything to be said about the qualitative nature of $S(a, x)$ ? Is it always a Borel set, measurable, $F_{\sigma}, G_{\sigma}$ ?

\section{REFERENCE}

1. WEISS, GARY "Commutators and operator ideals", dissertation, University of Michigan, 1975. 


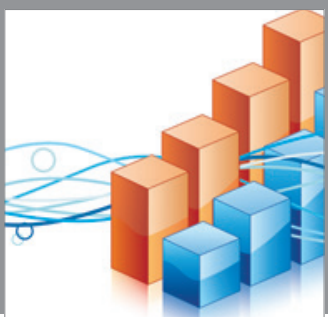

Advances in

Operations Research

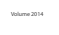

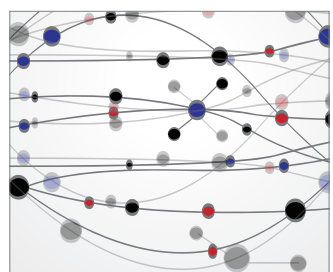

\section{The Scientific} World Journal
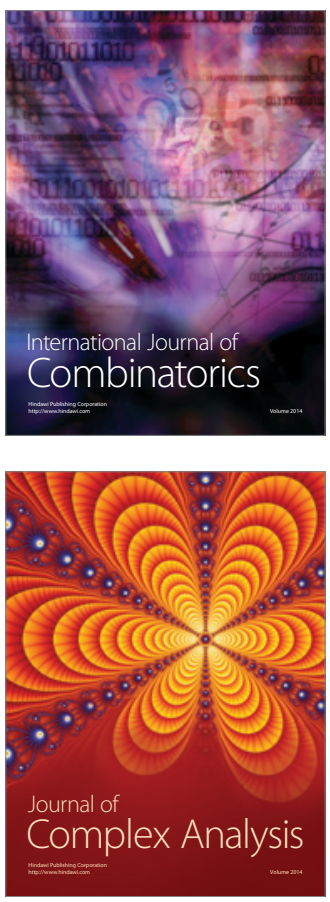

International Journal of

Mathematics and

Mathematical

Sciences
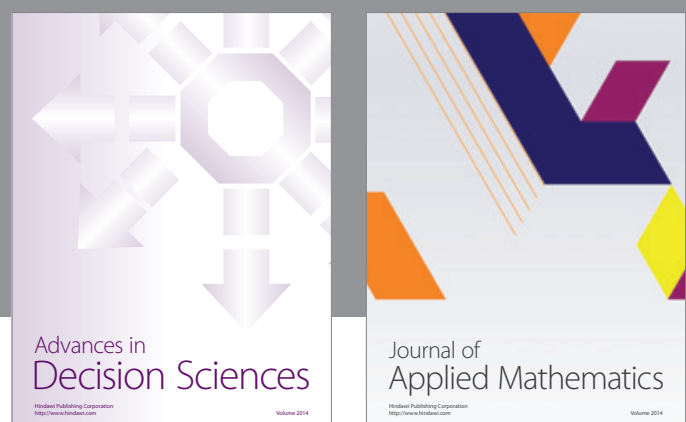

Journal of

Applied Mathematics
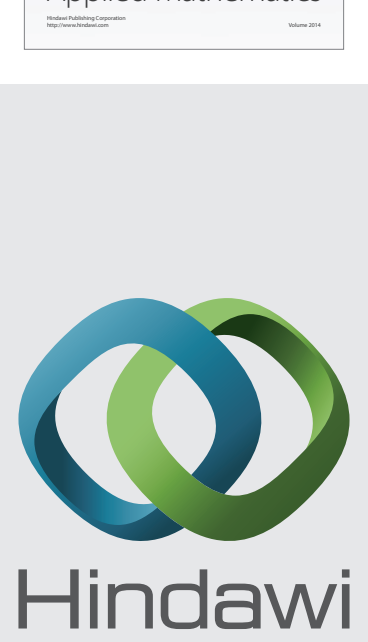

Submit your manuscripts at http://www.hindawi.com
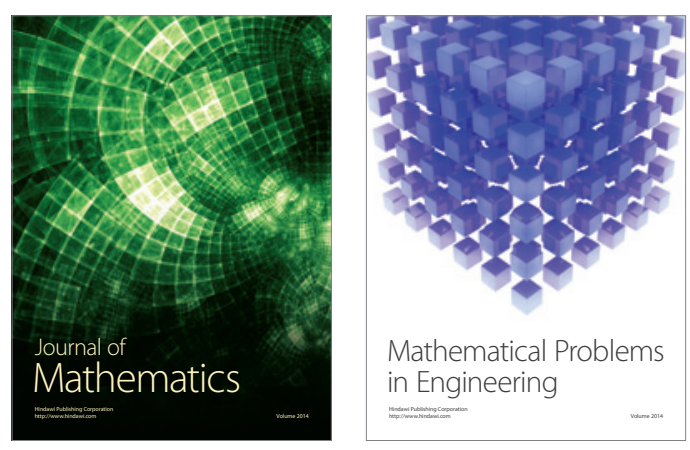

Mathematical Problems in Engineering
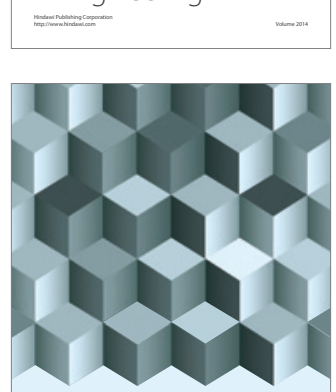

Journal of

Function Spaces
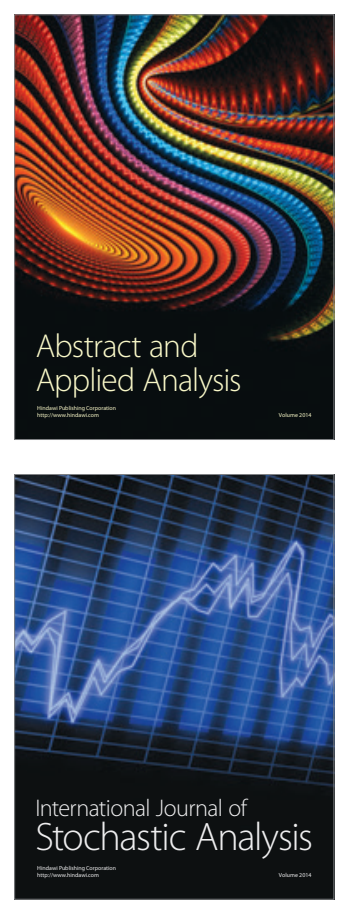

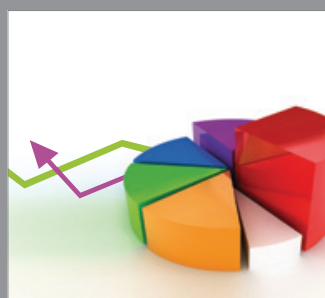

ournal of

Probability and Statistics

Promensencen
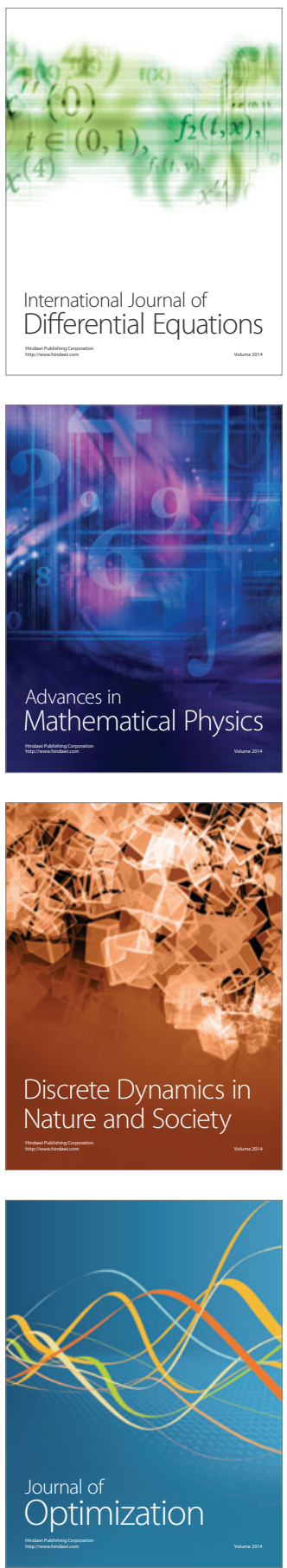